\title{
Detecting volcanic sulfur dioxide plumes in the Northern Hemisphere using the Brewer spectrophotometer, other networks, and satellite observations
}

Christos S. Zerefos ${ }^{1,2,3,10}$, Kostas Eleftheratos ${ }^{2,4}$, John Kapsomenakis ${ }^{1}$, Stavros Solomos ${ }^{5}$, Antje Inness ${ }^{6}$, Dimitris Balis ${ }^{7}$, Alberto Redondas ${ }^{8}$, Henk Eskes ${ }^{9}$, Vassilis Amiridis ${ }^{5}$, Christos Repapis $^{10}$, Marc Allaart ${ }^{9}$, Ronny Engelmann ${ }^{11}$, Arne Dahlback ${ }^{12}$, Veerle De Bock ${ }^{13}$, Henri Diémoz $^{14}$, Paul Eriksen ${ }^{15}$, Julian Gröbner ${ }^{16}$, Anu Heikkilä ${ }^{17}$, Janusz Jarosławski ${ }^{18}$, Weine Josefsson $^{19}$, Tomi Karppinen ${ }^{20}$, Ulf Köhler ${ }^{21}$, Charoula Meleti ${ }^{7}$, John Rimmer ${ }^{22}$, Vladimir Savinykh $^{23}$, Vadim Shirotov ${ }^{24}$, Anna Maria Siani ${ }^{25}$, Andrew R. D. Smedley ${ }^{22}$, Martin Stanek ${ }^{26}$, René Stübi ${ }^{27}$

${ }^{1}$ Research Centre for Atmospheric Physics and Climatology, Academy of Athens, Athens, Greece

${ }^{2}$ Biomedical Research Foundation, Academy of Athens, Athens, Greece

${ }^{3}$ Navarino Environmental Observatory (N.E.O.), Messinia, Greece

${ }^{4}$ Faculty of Geology and Geoenvironment, University of Athens, Greece

${ }^{5}$ Institute for Astronomy, Astrophysics, Space Applications and Remote Sensing (IAASARS), National Observatory of Athens, Athens, Greece

${ }^{6}$ European Centre for Medium-Range Weather Forecasts (ECMWF), Reading, UK

${ }^{7}$ Department of Physics, Aristotle University of Thessaloniki, Thessaloniki, Greece

${ }^{8}$ Izaña Atmospheric Research Center, AEMET, Tenerife, Canary Islands, Spain

${ }^{9}$ Royal Netherlands Meteorological Institute (KNMI), De Bilt, the Netherlands

${ }^{10}$ Mariolopoulos-Kanaginis Foundation for the Environmental Sciences, Athens, Greece

${ }^{11}$ Leibniz Institute for Tropospheric Research, Leibniz, Germany

${ }^{12}$ Department of Physics, University of Oslo, Oslo, Norway

${ }^{13}$ Royal Meteorological Institute of Belgium, Brussels, Belgium

${ }^{14}$ ARPA Valle d'Aosta, Saint-Christophe, Italy

${ }^{15}$ Danish Meteorological Institute, Copenhagen, Denmark

${ }^{16}$ PMOD/WRC, Davos Dorf, Switzerland

${ }^{17}$ Climate Change Unit, Finnish Meteorological Institute, Helsinki, Finland

${ }^{18}$ Institute of Geophysics, Polish Academy of Sciences, Warsaw, Poland

${ }^{19}$ Swedish Meteorological and Hydrological Institute, Norrköping, Sweden

${ }^{20}$ Arctic Research Centre, Finnish Meteorological Institute, Sodankylä, Finland

${ }^{21}$ DWD, Meteorological Observatory Hohenpeissenberg, Germany

${ }^{22}$ Centre for Atmospheric Science, School of Earth, Atmospheric and Environmental Sciences, University of Manchester, Manchester M13 9PL, UK

${ }^{23}$ A.M. Obukhov Institute of Atmospheric Physics, Kislovodsk, Russia

${ }^{24}$ Institute of Experimental Meteorology, Obninsk, Russia

${ }^{25}$ Department of Physics, Sapienza - University of Rome, Rome, Italy

${ }^{26}$ Solar and Ozone Observatory, Hradec Kralove, Czech Republic

${ }^{27}$ Federal Office of Meteorology and Climatology, MeteoSwiss, Payerne, Switzerland

Correspondence to: Christos S. Zerefos (zerefos@geol.uoa.gr) 
$\mathrm{SO}_{2}$ plume from Nabro Volcano over the Canary Islands (June 2011)

\section{1. $\mathrm{SO}_{2}$ algorithm improvement}

The $\mathrm{SO}_{2}$ is calculated routinely on the Brewer's ozone retrieval algorithm (Kerr et al., 1988).

$\mathrm{SO}_{2}=\mathrm{MS} 8-\mathrm{ETC} / \mathrm{m}_{3} \mathrm{~A} 3 * \mathrm{~A} 2-\mathrm{O}_{3} / \mathrm{A} 2$

Where:

MS8 is the linear combination of log count ratios of the $\mathrm{SO}_{2}$ wavelengths 306.3, 316.8, and $320.1 \mathrm{~nm}$, with weights $[-1.00,4.20,-3.20] ; \mathrm{m}_{3}$ is the ozone air mass factor; ETC is the extraterrestrial constant; and $\mathrm{A} 3$ and $\mathrm{A} 2$ are the effective cross sections of $\mathrm{SO}_{2}$. $\mathrm{A} 3$ is the differential $\mathrm{O}_{3}$ absorption coefficient for the $\mathrm{SO}_{2}$ wavelength combination whereas $\mathrm{A} 2$ is the ratio of the $\mathrm{SO}_{2}$ absorption coefficient to the $\mathrm{O}_{3}$ absorption coefficient with $\mathrm{SO}_{2}$ wavelength combination (A2 is nominally set equal to 2.44). These effective absorption cross section quantities can be measured during the wavelength characterization of the spectrometer using the same methodology as that used for the ozone absorption calculation (Redondas 2014). The $\mathrm{SO}_{2}$ cross section used by the Brewer network were calculated by Kerr, but certainly this subject needs more investigation as we cannot find the laboratory calibration to which is referenced this cross section.

The only calibration constant is the ETC. There are three methods to derive the ETC: 1) by comparing to a reference instrument, 2) assuming a zero $\mathrm{SO}_{2}$ during a certain period (this is what most of Brewer stations use to do), and 3) by Langley extrapolation. In this case analysis we show results using the last two methods, so the Langley extrapolation with the RBCC-E (Regional Brewer Calibration Centre for Europe) methodology (Redondas, 2007; Ito et al., 2011), and using the days 6 and 7 of June as zero reference. The estimated error of the Langley is +/- 0.25 DU. 


\section{Ancillary information}

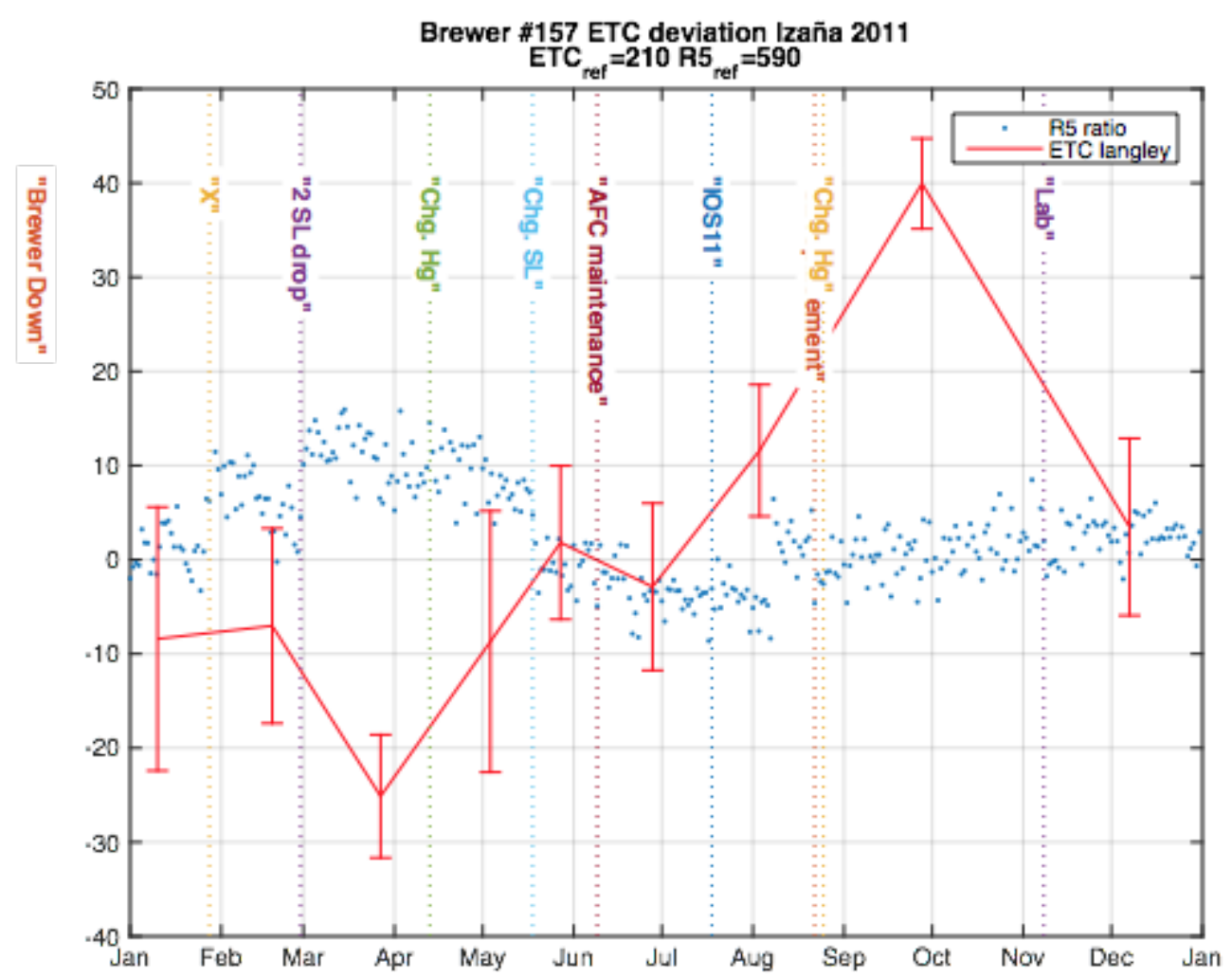

Figure S1: Langley determination of the $\mathrm{SO}_{2}$ extraterrestrial constant and the internal lamp measurement (R5) used for track the calibration between calibrations. Possible changes on the calibration of the instrument are marked by vertical lines. The error estimation of the ETC is shown on error bars and is in mean +/- 10 ETC units (+/- 0.25 DU).

\section{References}

Ito, M., Takano, M., Oguri, H., Takita, M., Shimodaira, H., and Ishitsuka, H.: Observations of total ozone and UV solar radiation with Brewer spectrophotometers on the Norikura mountains in 2009, Journal of the Aerological Observatory, 69, 41-54, 2011.

Kerr, J. B., Asbridge, I. A., and Evans, W. F. J.: Intercomparison of Total Ozone Measured by the Brewer and Dobson Spectrophotometers at Toronto, Journal of Geophysical Research, 93, 11129-11140, 1988.

Redondas, A.: Ozone absolute langley calibration, in The Tenth Biennial WMO Consultation on Brewer Ozone and UV Spectrophotometer Operation, Calibration and Data Reporting, Edited by C. T. McElroy and E. W. Hare, Gaw Report. No. 176, WMO TD No. 1420, pp. 12-14, 2007. 
Redondas, A., Evans, R., Stuebi, R., Köhler, U., and Weber, M.: Evaluation of the use of five laboratory-determined ozone absorption cross sections in Brewer and Dobson retrieval algorithms, Atmospheric Chemistry and Physics, 14(3), 1635-1648, 2014. 